\title{
IMPECCABLE: Integrated Modeling PipelinE for COVID Cure by Assessing Better LEads
}

\author{
Aymen Al Saadi ${ }^{1}$, Dario Alfe ${ }^{2,10}$, Yadu Babuji ${ }^{3}$, Agastya Bhati ${ }^{2}$, Ben Blaiszik ${ }^{3,4}$, Thomas Brettin ${ }^{4}$, \\ Kyle Chard ${ }^{3,4}$, Ryan Chard ${ }^{3}$, Peter Coveney ${ }^{* 2,9}$, Anda Trifan ${ }^{4}$, Alex Brace ${ }^{4}$, Austin Clyde ${ }^{2}$, Ian \\ Foster $^{3,4}$, Tom Gibbs ${ }^{8}$, Shantenu Jha*1,5, Kristopher Keipert ${ }^{8}$, Thorsten Kurth ${ }^{8}$, Dieter Kranzlmüller ${ }^{7}$, \\ Hyungro Lee ${ }^{1}$, Zhuozhao Li ${ }^{3}$, Heng Ma ${ }^{4}$, Andre Merzky ${ }^{1}$, Gerald Mathias ${ }^{7}$, Alexander Partin ${ }^{4}$, \\ Junqi Yin ${ }^{6}$, Arvind Ramanathan*4, Ashka Shah ${ }^{4}$, Abraham Stern ${ }^{8}$, Rick Stevens ${ }^{* 3,4}$, Li Tan ${ }^{5}$, Mikhail \\ Titov $^{1}$, Aristeidis Tsaris ${ }^{6}$, Matteo Turilli ${ }^{1}$, Huub Van Dam ${ }^{5}$, Shunzhou Wan ${ }^{2}$, David Wifling ${ }^{7}$ \\ ${ }^{1}$ Rutgers University, ${ }^{2}$ University College London, ${ }^{3}$ University of Chicago, ${ }^{4}$ Argonne National Laboratory, \\ ${ }^{5}$ Brookhaven National Laboratory, ${ }^{6}$ Oak Ridge Leadership Computing Facility, ${ }^{7}$ Leibniz Supercomputing Centre, \\ ${ }^{8}$ NVIDIA Corporation, ${ }^{9}$ University of Amsterdam, ${ }^{10}$ University of Naples Federico II, ${ }^{*}$ Contact Authors
}

\begin{abstract}
The drug discovery process currently employed in the pharmaceutical industry typically requires about 10 years and \$2-3 billion to deliver one new drug. This is both too expensive and too slow, especially in emergencies like the COVID-19 pandemic. In silico methodologies need to be improved to better select lead compounds that can proceed to later stages of the drug discovery protocol accelerating the entire process. No single methodological approach can achieve the necessary accuracy with required efficiency. Here we describe multiple algorithmic innovations to overcome this fundamental limitation, development and deployment of computational infrastructure at scale integrates multiple artificial intelligence and simulation-based approaches. Three measures of performance are: (i) throughput, the number of ligands per unit time; (ii) scientific performance, the number of effective ligands sampled per unit time; and (iii) peak performance, in flop/s. The capabilities outlined here have been used in production for several months as the workhorse of the the computational infrastructure to support the capabilities of the US-DOE National Virtual Biotechnology Laboratory in combination with resources from the EU Centre of Excellence in Computational Biomedicine.
\end{abstract}

\section{Keywords}

Datasets, neural networks, gaze detection, text tagging, docking molecular dynamics, free energy estimation

\section{ACM Reference Format:}

Aymen Al Saadi ${ }^{1}$, Dario Alfe ${ }^{2,10}$, Yadu Babuji ${ }^{3}$, Agastya Bhati ${ }^{2}$, Ben Blaiszik ${ }^{3,4}$, Thomas Brettin ${ }^{4}$, Kyle Chard ${ }^{3,4}$, Ryan Chard ${ }^{3}$, Peter Coveney ${ }^{* 2,9}$, Anda Trifan $^{4}$, Alex Brace ${ }^{4}$, Austin Clyde ${ }^{2}$, Ian Foster ${ }^{3,4}$, Tom Gibbs ${ }^{8}$, Shantenu $\mathrm{Jha}^{* 1,5}$, Kristopher Keipert ${ }^{8}$, Thorsten Kurth ${ }^{8}$, Dieter Kranzlmüller ${ }^{7}$, Hyungro Lee ${ }^{1}$, Zhuozhao $\mathrm{Li}^{3}$, Heng $\mathrm{Ma}^{4}$, Andre Merzky ${ }^{1}$, Gerald Mathias ${ }^{7}$, Alexander Partin ${ }^{4}$, Junqi Yin ${ }^{6}$, Arvind Ramanathan ${ }^{* 4}$, Ashka Shah ${ }^{4}$, Abraham Stern ${ }^{8}$, Rick Stevens ${ }^{* 3,4}$, Li Tan $^{5}$, Mikhail Titov ${ }^{1}$, Aristeidis Tsaris ${ }^{6}$,

Permission to make digital or hard copies of all or part of this work for personal or classroom use is granted without fee provided that copies are not made or distributed for profit or commercial advantage and that copies bear this notice and the full citation on the first page. Copyrights for components of this work owned by others than ACM must be honored. Abstracting with credit is permitted. To copy otherwise, or republish, to post on servers or to redistribute to lists, requires prior specific permission and/or a fee. Request permissions from permissions@acm.org.

Supercomputing '20, November 16-19, 2020, Virtual

(C) 2018 Association for Computing Machinery.

ACM ISBN 978-x-xxxx-xxxx-x/YY/MM. . . \$15.00

https://doi.org/10.1145/1122445.1122456
Matteo Turilli ${ }^{1}$, Huub Van Dam ${ }^{5}$, Shunzhou Wan ${ }^{2}$, David Wifling ${ }^{7} .2020$. IMPECCABLE: Integrated Modeling PipelinE for COVID Cure by Assessing Better LEads. In Supercomputing '20: International Conference for High Performance Computing, Networking, Storage, and Analysis. ACM, New York, NY, USA, 13 pages. https://doi.org/10.1145/1122445.1122456

\section{Justification}

COVID-19 has claimed a million lives and resulted in over 35 million infections; there is an urgent need to identify drugs that can inhibit SARS-CoV-2. IMPECCABLE innovatively couples multiple algorithms to overcome fundamental limitations of classical in silico drug design. We discuss how algorithmic and computational innovations are advancing scientific discovery.

\section{Performance Attributes}

Performance attributes are listed in Table 1.

Table 1: Performance Attributes

\begin{tabular}{ll}
\hline Performance Attribute & Our Submission \\
\hline \hline Category of achievement & $\begin{array}{l}\text { Scalability, time-to-solution, } \\
\text { peak performance }\end{array}$ \\
Type of method used & Explicit \\
Results reported on basis of & Whole app including I/O \\
Precision reported & Mixed \\
System scale & Measured on full system \\
Measurement mechanism & Timers, FLOP count, \\
& performance modeling \\
\hline
\end{tabular}

\section{Problem Overview}

Drug discovery is an astonishingly resource intensive process; the average time to search, design, and effectively bring a clinically tested drug can range between 10 to 15 years, and can cost over 1 billion dollars [25, 39]. Considering the universe of about $10^{68}$ possible compounds to traverse for effective drugs, there is an immediate need for more efficient, higher throughput, and more meaningful frameworks for early stage drug discovery [15].

In the context of COVID-19, a grand challenge within the drugdiscovery community is the need for capabilities that can screen tens of billions of small molecules against the SARS-CoV-2 proteome and identify high quality lead molecules that have the potential to inhibit the virus life cycle [34]. To achieve this goal, in silico 
methodologies need to be significantly improved to better design lead compounds that have the potential to become drugs that can be pushed to the later stages of drug discovery [9, 40, 61]. However, no single algorithm or method can achieve the necessary accuracy with required efficiency. IMPECCABLE innovatively brings together multiple algorithms into a single unified pipeline with an interactive and iterative methodology allowing both upstream and downstream feedback to overcome fundamental limitations of classical in silico drug design. We demonstrate the impact of IMPECCABLE by measuring both raw throughput, defined as ligands per unit time, as well as its scientific performance, defined as ligands sampled per unit time.

\subsection{Targeting the SARS-CoV-2 proteome}

The SARS-CoV-2 genome consists of 29 proteins of which 16 nonstructural proteins (NSPs) represent various enzymes that play critical roles in the virus life cycle [34]. In spite of our understanding of the roles of these NSPs in the virus life cycle as well as the fact that closely related protein structures from the middle eastern respiratory syndrome (MERS) and the SARS coronaviruses are known, there are no known antivirals currently available for SARS-CoV-2 [51] The structural biology community has embarked on a massive effort to provide access to 3D crystallographic structures of the NSPs and therefore, this represents a tremendous opportunity for designing therapeutics targeting the disease.

Early stages of drug discovery rely on (experimental) high throughput screening (HTS) protocols to hone in on a suitable chemical compound library that can be particularly useful against known protein targets [16]. Although HTS approaches are widely available and used, the sheer combinatorics of drug-like molecules poses a tremendous challenge in exhaustively sampling the compound space to find viable molecules. Instead, a number of open source initiatives are currently building virtual HTS platforms targeting the entire viral proteome [1, 2]. Indeed, the goals of our efforts include high throughput structure-based protein-ligand docking simulations, followed by iterative refinements to these virtual screening results to filter out compounds that "show promise" in biochemical or wholecell assays, safety and toxicology tests, finally leading to a set of compounds that can proceed towards clinical trials. This process represents a filtering process, with successive steps leading to better drug candidates targeting the virus. Although covering such a vast chemical space would be nearly impossible, even with access to the entire world's computing resources, there are open data resources which provide access to a fairly representative, yet diverse chemical space $\left(\sim 10^{12}\right.$ compounds).

\subsection{The IMPECCABLE Solution}

Artificial intelligence (AI) and machine learning (ML) have played a pivotal role in COVID-19 drug discovery [61]. However, most ML/AI efforts have largely focused on building effective means to analyze large volumes of data generated through either ligand docking simulations-for the purposes of filtering favorable vs. unfavorable ligand binding poses in a given protein-or molecular dynamics (MD) simulations of selected protein-ligand complexes.

While docking programs are generally good at pose prediction, they are less effective in predicting binding free-energy of proteinligand complexes. Similarly, while MD simulations are effective at predicting binding-free energies, their intrinsic limitations in sampling protein-ligand complex formation processes imply that the approach may be computationally infeasible to translate on large compound libraries. If ML/AI methods can glue information across docking and MD simulation techniques, while leveraging their individual strengths to provide meaningful feedback in terms of identifying parts of the compound libraries that may result in better lead molecules, higher effective throughput (sampling larger compound libraries), and meaningful enrichment of lead molecules for targeting SARS-CoV-2 proteins would be possible.

IMPECCABLE addresses these scientific and methodological challenges by providing a scalable, flexible, and extensible infrastructure for campaigns designed to discover improved leads targeted at SARS-CoV-2. As shown in Fig. 1, the IMPECCABLE campaign consists of an iterative loop initiated with ML predictions (ML1), followed by three stages of data processing (S1, S2, S3). IMPECCABLE centers on the use of ML/AI techniques (ML1 and S2) interfaced with physics-based computational methods to estimate docking poses of compounds that are promising leads for a given protein target (S1) and binding free-energy computations (S3).

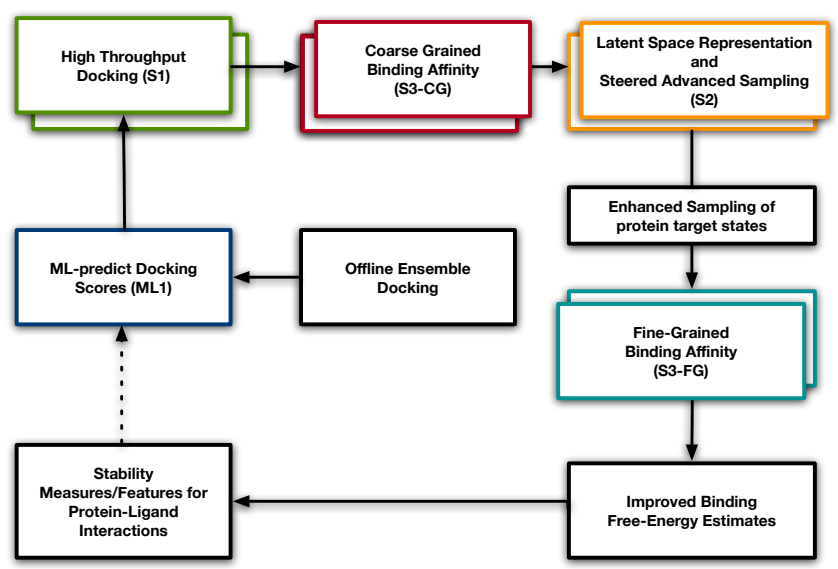

Figure 1: The IMPECCABLE Solution: represents an entire virtual drug discovery pipeline, from hit to lead through to lead optimization. The constituent components are deep-learning based surrogate model for docking (ML1), Autodock-GPU (S1), coarse and fine-grained binding free energies (S3-CG and S3FG) and S2 (DeepDriveMD).

ML techniques overcome the limitations of S1 and S3 by predicting the likelihood of binding between small molecules and a protein target (ML1), and accelerating the sampling of conformational landscapes to bound the binding free-energy values for a given protein-ligand complex (S2). Interfacing ML approaches with physics-based models (docking and MD simulations), we achieve at least three orders of magnitude improvement in the size of compound libraries that can be screened with traditional approaches, while simultaneously providing access to binding free-energy calculations that can impose better confidence intervals in the ligands selected for further (experimental or computational) optimization. ML1: Machine Learning Models for docking score prediction Scoring functions are used to score poses in order to determine the most likely pose of the molecule, the magnitude of which is used 
to provide an indication of active versus inactive ligands, and lastly to rank order sets of libraries. We create a ML surrogate model to replace the use of docking as a means of locating regions of chemical space likely to include strong binding drug leads. The only free variable for the surrogate ML ranking function is the basic molecular information, which typically presents as a SMILES string. We use a simple featurization method, namely 2D image depictions, as they do not require complicated architectures such as graph convolution networks, while demonstrating good prediction. We obtain these image depictions from the nCov-Group Data Repository [10], which contains various descriptors for 4.2B molecules generated on HPC systems with the assistance of Parsl [11].

S1: High-throughput Docking Protein-ligand docking encompasses a computational pipeline consisting of ligand 3D structure (conformer) enumeration, exhaustive docking and scoring, and final pose scoring. The input to the docking protocol requires a protein structure with a designed binding region, or a crystallized ligand from which a region can be inferred, as well as a database of molecules to dock in SMILES format. SMILES format is a compact representation of a 2D molecule.

S2: Machine Learning Driven Molecular Dynamics Machine learning tools are able to quantify statistical insights into the timedependent structural changes a biomolecule undergoes in simulations, identify events that characterize large-scale conformational changes at multiple timescales, build low-dimensional representations of simulation data capturing biophysical/biochemical/biological information, use these low-dimensional representations to infer kinetically and energetically coherent conformational substates, and obtain quantitative comparisons with experiments.

Deep structured learning approaches automatically learn lowerlevel representations (or features) from the input data and successively aggregating them such that they can be used in a variety of supervised, semi-supervised and unsupervised machine learning tasks. We developed variational autoencoders to automatically reduce the high dimensionality of MD trajectories and cluster conformations into a small number of conformational states that share similar structural, and energetic characteristics [14]. We use S2 to drive adaptive sampling simulations, and use the acceleration of "rare" events, to investigate protein-ligand interactions [28, 37].

S3: Binding Free Energy Calculations Hit-to-Lead (H2L), sometimes also called lead generation, is a step in the drug discovery process where promising lead compounds are identified from initial hits generated at preceding stages. It involves evaluation of initial hits followed by some optimization of potentially good compounds to achieve nanomolar affinities. The change in free energy between free and bound states of protein and ligand, also known as binding affinity, is a promising measure of the binding potency of a molecule, and hence it is used as a parameter for evaluating and optimizing hits at H2L stage. We employ the ESMACS protocol [47-49, 55, 56], for estimating binding affinities of protein-ligand complexes. We differentiate between coarse-grained (CG) and fine-grained (FG) ESMACS variants, which differ in the number of replicas (6 vs. 24), equilibration duration ( 1 vs. $2 \mathrm{~ns}$ ), simulation duration ( 4 vs. 10ns) etc. The computational cost of ESMACS-CG is about an order of magnitude less than that of ESMACS-FG.

IMPECCABLE integrates multiple methods and dynamically selects active ligands for progressively expensive methods. In fact, at any stage, only the most promising candidates are advanced to the next stage, yielding a $\mathrm{N}$-deep pipeline, where each downstream stage is computationally more expensive, but also more accurate than previous stages. The methods chosen vary in computational cost per ligand by more than six orders of magnitude; in the docking stage of IMPECCABLE each dock costs about $10^{-4}$ node-hours per ligand; fine-grained binding free energy costs about $10^{2}$ node-hours per ligand. This provides an important dynamic range of accuracy, and thus potential for scientific performance enhancement. Tuning the cost of each method by extending or contracting the number of iterations of each method allows for enhanced scientific performance and throughput.

The integration of methods with varying computational characteristics into a cohesive whole to support sustained computational campaign requires innovative computational infrastructure. There are no turnkey or shrink-wrap solutions to support campaigns. We employ RADICAL-Cybertools (RCT) [36], a set of software systems, to manage the execution of heterogeneous workflows and workloads on leadership computing facilities. RCT have the required design and necessary abstractions to manage the heterogeneity of workloads and platforms, and the integrated campaign.

\section{State of the Art}

We outline briefly the current state-of-the-art with respect to our computational campaign, summarizing some of the challenges and limitations in the context of IMPECCABLE .

The idea of building large-scale virtual screening pipelines is not entirely new. However, many of these toolkits are either proprietary (e.g., Schrodinger, OpenEye) or even if they are openly available (e.g., AMPL [32]), they end up being very customized. Some of the early approaches $[26,41,60]$ proposed the use of statistical techniques to be incorporated - either for downstream refinement or for selection of viable leads - however, sheer size of ligand docking simulations as well as MD simulations could easily overwhelm these approaches, leading to very little gain from the overall process. With the advent of deep learning [27] and recent advances in computing technology for running MD simulations, the burden of handling large volumes of virtual screening data and simulations has been largely reduced. This in turn has spurred momentum in developing several automated pipelines whose goal is to improve not just the virtual screening process itself, but also to enable downstream processes such as lead refinement and optimization.

Central to most high throughput virtual screening pipelines are scoring functions that are used to determine which pose is selected via exhaustive search and how the resulting molecule and pose rank within a dataset. While some docking protocols expand the typical exhaustive mapping of a scoring function over possible positions, the distinction between protocols boils down to the scoring function.

The scoring function is used for three purposes: (i) to detect when the ligand is properly positioned in the pocket (pose prediction); (ii) to provide a general assessment of activity (virtual screening), and (iii) to rank ordering compound libraries from their selected poses (binding affinity ranking) [18, 23]. Given the central role of scoring functions, the enterprise of molecular docking pragmatically and theoretically rests on the speed and accuracy of these scoring functions. Some scoring functions can be slow, and particularly well suited for pose prediction, while others may be fast and only capable 
of decoy detection. However, scoring functions by themselves are not sufficient; they need to be augmented with physically accessible (and experimentally verifiable) quantities such as binding free-energies (or binding affinities).

There are a large number of in silico methods available to calculate binding affinities. The major ones, in the order of increasing accuracy as well as computational expense, are as follows: (1) molecular docking [13, 33], (2) linear interaction energy [63], (3) MMPBSA/MMGBSA and (4) alchemical methods (Thermodynamic Integration/Free Energy Perturbation) [43, 62]. Alchemical methods are theoretically the most exact whereas the other three involve approximations to some extent, decreasing in the above order. This is why we need to choose an appropriate method for each step in the entire drug discovery process, keeping in mind the level of accuracy desired and the number of compounds to be considered. In our workflow, we employ the cheapest and not greatly accurate docking methods at the initial stages, MMPBSA-based ESMACS at the hit-to-lead stage and TI based TIES at the lead optimization stage. The throughput reduces by orders of magnitude as we move to subsequent stages with higher levels of accuracy desired and hence the computational cost remains under control.

As demonstrated in Tab. 2, IMPECCABLE integrates methods with 6-7 orders of magnitude computational cost, and although hitherto not part of our demonstrated scientific impact, can support TIES which is a further two orders of magnitude more expensive than ESMACS. Thus, IMPECCABLE represents a unique solution that integrates multiple methods, with a collective and integrated performance significantly greater than any single algorithm and method alone.

\section{Innovations Realized}

IMPECCABLE embodies innovation within the individual methods it employs, as well as in the way it couples these methods. Underpinning this coupling is a flexible and scalable infrastructure. The ability to potentially screen much larger libraries with higher throughputs so as to identify greater number of of viable SARS-CoV-2 protein target specific leads is the ultimate measure of scientific productivity. To that end, we characterize the contribution of each component, as well as the improvement in performance of the IMPECCABLE pipeline by integrating individual components. Actual results are presented in Section 7.

\subsection{Algorithmic \& Methodological}

5.1.1 S1: AutoDock-GPU The CUDA-accelerated AutoDock4.2.6 (AutoDockGPU) leverages a highly parallel implementation of the Lamarckian genetic algorithm (LGA) by processing ligand-receptor poses in parallel over multiple compute units. AutodockGPU was developed in collaboration with NVIDIA and Aaron Scheinberg (Jubilee Development) with a target of the Summit system at Oak Ridge Leadership Computing Facility (OLCF). AutoDockGPU applies the legacy Solis-Wets local search method along with a new local-search method based on gradients of the scoring function. One of these methods, ADADELTA, has proven to increase significantly the docking quality in terms of RMSDs and scores with observed speedups of 56x over the original serial AutoDock 4.2 (Solis-Wets) on CPU. A drug screen takes the best scoring pose from these independent outputs. Autodock-GPU uses OpenMP threading-based pipeline for hiding ligand input and staging, and the receptor-reuse functionality for docking many ligands to a single receptor. From the computational performance perspective, we measure the total number of docking calculations performed per GPU, which provides a measure of the overall docking capability.

5.1.2 ML1: ML-based docking score predictor We developed a ML surrogate model to replace docking as means of locating regions of chemical space likely to include strong binding drug leads. Docking does not rely on proteins, instead proteins are coded into the scoring function. Thus the only free variable for the surrogate ML ranking function is the basic molecular information. This usually presents as SMILES string, and there is an entire field of deep learning for molecular property prediction based on this approach [21].

A simple featurization method has been widely ignored-2D image depictions. From the 2D depiction of a molecule, chemists are generally able to identify major properties such as H-acceptors, estimate the molecule's weight, and even determine if a molecule might bind to a protein $[22,35,57]$. This featurization method, unlike graph structure, is able to utilize off-the-shelf convolutional neural networks. By using 2D images, we are able to initialize our models with pretrained weights that are typically scale and rotation invariant for image classification tasks, which is exactly we require in order to infer if a small molecule will bind well to a given SARSCoV-2 target.

We model the gains and losses using a cost function based on the regression enrichment surface (RES)[18]. The RES measures how well a surrogate model can detect the true top ranking molecules given a certain allocation of predicted hits.

While this analysis brings forth the failure of the model to exactly replicate the rank ordering of the compounds at scale, it provides the operational benefit of these models-the predictive ML model will indeed be able to filter with near $100 \%$ accuracy two orders of magnitude from the data library. Thus, if all else is equal, with only the additional cost of docking additional compounds, we are able to expand the set of viable leads detected by two orders of magnitude, without loss of performance in the top regions of detection (Sec. 7). 5.1.3 S3: Adaptive ESMACS Binding affinity is a small number (a few tens of $\mathrm{kcal} / \mathrm{mol}$ ) that is derived from absolute free energies which are large (a few hundreds to thousands of $\mathrm{kcal} / \mathrm{mol}$ ). Thus, the usual practice of performing MMPBSA calculations on conformations generated using a single MD simulation does not give reliable binding affinities. ESMACS, on the other hand, performs ensemble MD simulation, where each independent simulation is termed a replica. Parameters such as the size of ensemble simulation (or the number of replicas) and the length of individual replica are chosen such that our results become reliable quantities [38, 54]. Another factor that plays a role in determination of these parameters is the level of precision desired and the cost-benefit ratio. The number of replicas performed is adjusted to a find a sweet spot between computational cost and the level of precision acceptable at a particular stage of the pipeline.

ESMACS is costlier than the standard approach of performing a single simulation of similar duration. This increased cost however, is more than compensated by the enhanced precision of ESMACS results which makes the resultant ranking of compounds much more reliable compared to standard approaches with similar accuracy. 
MMPBSA based free energies have huge variability in results rendering them non-reproducible [38, 47, 49, 50, 54]. In fact, fewer iterations are required to achieve the same level of convergence in chemical space on using ensemble simulation based methods, which leads to comparable (or even reduced) computational cost overall, than on using standard single simulation approaches. This apparent increased cost has advantages, such as an increased level of confidence in predicted ranking of compounds, and thus much more reliable training data for an ML model.

We used ESMACS-CG to perform the initial screening of thousands of hits in order to reduce computational cost while compromising on the level of precision and ranking of compounds, and used ESMACS-FG for the latter stages when we have better binding poses, and/or LPC conformations. Selectively using ESMACS-FG on a refined set of complexes decreases the computational cost substantially without affecting the quality of results.

5.1.4 S2: Al-Driven MD We leverage DeepDriveMD [29] to simulate large ensembles of protein-ligand complexes. We have shown that DeepDriveMD can potentially accelerate protein folding simulations by at least 2 orders of magnitude. Here DeepDriveMD (S2) builds an adaptive sampling framework to support the exploration of protein-ligand bound states that are not often accessible to approaches such as ESMACS (S3).

A key innovation is support for extremely large numbers of ligandprotein complexes (LPC). This stems from the fact that a ESMACSCG (S3-CG) simulations may generate on average, six ensembles which are analyzed by our novel MD-driven AI approaches to identify 5-10 novel states. Hence, we also implemented a novel approach for analyzing large MD ensemble simulation datasets using a 3D adversarial autoencoder (3D-AAE), a significant improvement over approaches such as variational autoencoders in that it is more robust and generalizable to protein coordinate datasets than contact maps (or other raw inputs) extracted from MD simulations. Similarly to autoencoders, 3D-AAE builds a latent embedding space for MD simulations to characterize conformational changes within protein-ligand complexes from ESMACS-CG/FG simulation trajectories. The 3D-AAE includes the PointNet encoder, Chamfer distance-based reconstruction loss, and a Wasserstein adversarial loss with gradient penalty to build a latent manifold on which all simulations are projected. From this latent manifold, we use local outlier factor (LOF) detection to identify 'interesting' protein-ligand complexes that are then selected for S3-FG simulations. The iterative feedback between two stages of S3-CG/FG and S2 enables accurate estimates for the binding free-energy, and allows us to filter compounds based on their affinity to the protein, while accounting for the intrinsic conformational flexibility of the LPC.

Measuring DeepDriveMD performance for LPCs presents additional challenges. For example, input from the S3-CG pipeline stage are relatively short time-scale, whereas LPC association processes tend to vary significantly in time scales. Thus, we chose a pragmatic measure of LPC stability that takes into account the number of heavy atom contacts between the protein and the ligand of interest. From the top scoring LPCs that are selected from S3-CG, we use a novel 3D-AAE to filter those conformations that show increased stability profiles in the LPCs. We posit that these LPCs are of the most interest, since the increased stability potentially contributes to favorable interactions between protein and ligand. We also measure 3D-AAE performance in terms of its ability to learn effective latent space representations from S3-CG stage (through standard measures such as training and validation loss metrics).

Table 2: Normalized computational costs on Summit.

\begin{tabular}{l|rrr}
\hline Method & $\begin{array}{c}\text { Nodes per } \\
\text { ligand }\end{array}$ & $\begin{array}{c}\text { Hours per } \\
\text { ligand } \\
\text { (approx) }\end{array}$ & $\begin{array}{c}\text { Node-hours } \\
\text { per ligand }\end{array}$ \\
\hline \hline Docking (S1) & $1 / 6$ & 0.0001 & $\sim 0.0001$ \\
BFE-CG (S3-CG) & 1 & 0.5 & 0.5 \\
Ad. Sampling (S2) & 2 & 2 & 4 \\
BFE-FG (S3-FG) & 4 & 1.25 & 5 \\
BFE-TI (not integrated) & 64 & 10 & 640 \\
\hline
\end{tabular}

Putting it together and collective performance. Each stage of IMPECCABLE when augmented with relatively simple ML/AI approaches provides a significant boost to the coverage of the compound diversity as well as conformational landscapes of proteinligand complexes. Using training data generated on small $\mathrm{O}\left(10^{6}\right)$ compound libraries, ML1 enables a significant improvement in filtering large compound $\mathrm{O}\left(10^{9}\right)$ libraries, increasing the coverage by 4-6 orders of magnitude.

The second step, which results in filtering the top $1 \%$ of these compounds (which can also set by the end-user) through AutoDockGPU, identifies high confidence lead molecules that can bind to a given SARS-CoV-2 target. The purpose of the ML1 is to predict if the given molecule will dock the protein well, and not to predict the docking pose. We exploit the intrinsic strengths of most docking programs in predicting the binding pose for a given LPC, such that the intial poses selected follow physical principles (i.e., optimizing electrostatic and hydrophobic complementarity).

The next stage, namely S3-CG refines the filtered compounds to obtain an estimate of the binding-free energy. This step is crucial in the sense that it seeds the further pipeline with higher confidence leads that may have favorable interactions with the protein target.

This set of diverse LPC are input to S2, which leverages the 3DAAE to learn a latent manifold that consists of a description of which LPCs are most stable. In addition, the latent manifold also captures intrinsic dimensions of the protein's conformational landscape that are perturbed by the ligand's interactions. Using outlier detection methods, we then filter these landscapes further to include only a small number of LPCs on which SG-FG are implemented to ultimately suggest strong confidence intervals for binding free-energy of the LPCs selected.

The final stage of the pipeline provides additional features that identify key complementarity features (e.g., electrostatic interactions through hydrogen bonds or hydrophobic interactions) that can be input to the docking program for further refinement. Each successive iteration of IMPECCABLE thus provides successive yields of LPCs that could be modeled as an active learning pipeline for obtaining highly specific small-molecules that can inhibit a SARS-CoV-2 protein of interest. 


\subsection{Computational Infrastructure}

The integration of aforementioned diverse methods with varying computational characteristics, performance and scalability challenges, into a dynamic and adaptive computational campaign requires innovative computational infrastructure. The campaign workload is a diverse mix of task types, e.g., MPI, single GPU, multinode GPU and regular CPU; this mix of tasks changes over the course of the campaign There are multiple stages which couple and concurrently execute deep learning and traditional simulations. Coupling and concurrently executing these diverse tasks is challenging, but is made more difficult by virtue of having different models and coupling with simulations across multiple stages.

The dynamic variation of workload arises due to many reasons, for example: (i) adaptive methods, e.g., each LPC has a different rate of convergence for structural and energetic properties, and thus the duration varies; (ii) cost of docking per ligand varies across different drug compound libraries and the ligands they contain; and (iii) for methods that involve learning, (re-) training times are dependent on specific ligands and the number of simulations.

IMPECCABLE employs the Ensemble Toolkit (EnTK)[12], which uses RADICAL-Pilot (RP) [31] for flexible and scalable execution of workflows with heterogeneous tasks. Together they conform to the middleware building blocks architectural pattern — which constitute recent advances in the science of HPC workflows [44] — to permit a decoupling of the programming system from underlying execution capabilities.

5.2.1 Programming System EnTK is a Python implementation of a workflow engine, designed to support the programming and execution of applications with ensembles of tasks. EnTK executes tasks concurrently or sequentially, depending on their arbitrary priority relation. We use the term "task" to indicate a stand-alone process that has well-defined input, output, termination criteria, and dedicated resources. For example, a task can indicate an executable which performs a simulation or a data processing analysis, executing on one or more nodes on Summit. Tasks are grouped into stages and stages into pipelines depending on the priority relation among tasks. Tasks without a reciprocal priority relation can be grouped into the same stage, whereas tasks that need to be executed before other tasks have to be grouped into different stages. Stages are then grouped into pipelines and, in turn, multiple pipelines can be executed either concurrently or sequentially. Specifically, EnTK:

- permits asynchronous execution of concurrent pipelines (each pipeline can progress at its own pace);

- allows arbitrary sizing of stages (variable concurrency);

- supports heterogeneous tasks of arbitrary types, and combinations, as well as their inter-mixing;

- promotes "ensembles" as first-class code abstraction;

- selects parameters at runtime so as to provide near-optimal selection of cost versus accuracy [19, 20].

These are necessary capabilities to explore LPCs of varying complexity and cost, without constraining the number of concurrent investigations, and different methods run in arbitrary order.

5.2.2 Execution Framework for Dynamic Resource Management Given the extreme workload heterogeneity and workload variation between and across stages, dynamic resource management is critical. Dynamic resource management capability is provided by
RADICAL-Pilot (RP) [31, 45], a Python implementation of the pilot paradigm and architectural pattern [46]. Pilot systems enable users to submit pilot jobs to computing infrastructures and then use the resources acquired by the pilot to execute one or more workloads, i.e., set of tasks. Tasks are executed concurrently and sequentially, depending on the available resources. For example, given 10,000 single-node tasks and 1000 nodes, a pilot system will execute 1000 tasks concurrently and each one on the remaining 9000 tasks sequentially, whenever a node becomes available. RP enables the execution of heterogeneous workloads comprised of one or more scalar, MPI, OpenMP, multi-process, and multi-threaded tasks. RP directly schedules and executes on the resources of one or more pilots without having to use the infrastructure's batch system.

RP offers unique features when compared to other pilot systems or tools that enable the execution of multi-task workloads on HPC systems: (1) concurrent execution of heterogeneous tasks on the same pilot; (2) support of all the major HPC batch systems; (3) support of more than twelve methods to launch tasks; and (4) a general purpose architecture. RP can execute single or multi core tasks within a single compute node, or across multiple nodes. RP isolates the execution of each tasks into a dedicated process, enabling concurrent execution of heterogeneous tasks by design.

\section{Performance Measurement}

To understand the performance of IMPECCABLE it is imperative to understand the nature of the computational campaign, its composite workflows, their constituent workloads, desired performance and factors determining scalability.

\subsection{Computational Characteristics}

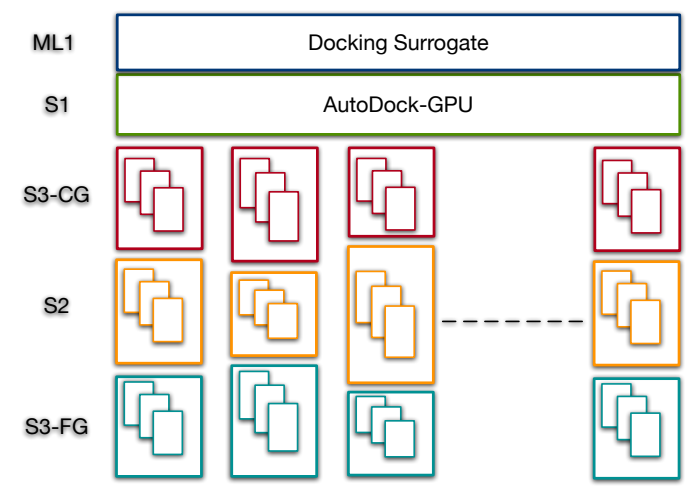

Figure 2: Programming and execution view: Each stage of the (S3-CG)-(S2)-(S3-FG) pipeline comprises multiple heterogeneous tasks; each stage executes for varying durations.

Fig. 2 provides an overview of how the IMPECCABLE campaign is constructed and executed. It comprises four distinct computational workflows: a machine learning surrogate (ML1), docking (S1), binding free energy calculations (S3), and latent space representation and steered advanced sampling via MD simulations (S2). Each is a distinct workflow with well-defined inputs and outputs, multiple executables with defined dependencies, and termination criteria, able to produce stand-alone scientifically meaningful end-results. Each workflow represents the expertise and unique scientific and methodological contribution from a different team. 
We codify IMPECCABLE workflows as a five-stage EnTK pipeline using a general-purpose language (Python) and application-specific constructs from the PST (Pipeline, Stage, Task) programming model. These abstraction simplify creating and executing ensemble applications with complex coordination and communication requirements. Pipelines can contain different workloads, e.g., distinct instances of $\mathrm{S}^{*}$ for a given LPC, but also possibly multiple instances of a given $\mathrm{S}^{*}$ for many LPC concurrently. Autodock-GPU is executed as a single task running on several thousand nodes, as is the docking surrogate, which is a relatively short duration task. The remaining three stages are workflows which are expressed as pipelines, comprised of differing stages and varying duration and number of tasks concurrently executing. The horizontal length of a box is proportional to the number of nodes utilized by a stage / computation, and the vertical length of boxes represent the temporal duration; boxes are not drawn to scale (see Table).

6.1.1 ML1: Deep Learning Docking Emulator This step is a docking emulator which serves as a pre-selection tool for docking calculations performed in step S1. The goal is to reduce the search space from about $126 \mathrm{M}$ ligands down to a manageable amount for the docking calculations. The emulator is based on a resnet-50 [24] deep neural network: it transforms image representations of ligand molecules into a docking score. To convert the ligand SMILES strings into images we employed the mol2D drawing submodule from rdKit [7]. The target scores are binding energies which are mapped into the interval $[0,1]$, with higher scores representing lower binding energies and thus higher docking probabilities. The main computational motifs are dense linear algebra, convolutions and elementwise operations on 4D tensors. The network is implemented in PyTorch and pre-trained on 500,000 randomly selected samples from the OZD ligand dataset across each receptor (for our purposes, each PDB entry corresponds to a separate receptor, providing access to an ensemble of docking simulations). For deployment, we compiled the model using NVIDIA TensorRT v7.2 [6] with cuDNN v8.0 [17] employing the torch2trt helper tool [8]. As base precision we chose half precision (FP16), so that we can use the Tensor Cores on Summit's V100 GPUs.

Inference workloads are notoriously IO bound, and thus we employ various optimizations to improve throughput. We start with the ULT911 dataset [5], which is supplied as a collection of 12,648 files with 10,000 ligands, each in Python pickle format. We first used gzip to compress each file, achieving an average compression factor of 14.2. We use MPI to distribute the individual files evenly across a large number of GPUs and bind one rank to each GPU. While we perform the model scaffolding phase, i.e. creating the computational graph and loading the weights from the pre-trained model file, each rank stages its assigned shard of the data from GPFS into node-local NVME. During the inference process, each rank utilizes multiple data loader processes where each is employing 2 prefetching threads: the first one loads compressed files from NVME into DRAM and decompresses them on the fly while the second iterates through the uncompressed data in memory, extracts the image and metadata information and feeds them to the neural network. The whole logic is implemented using the thread-safe python queue module. We further use careful exception handling to make the setup resilient against sporadic IO errors. After inference is done, the resulting lists of docking scores and metadata information such as ligand id and

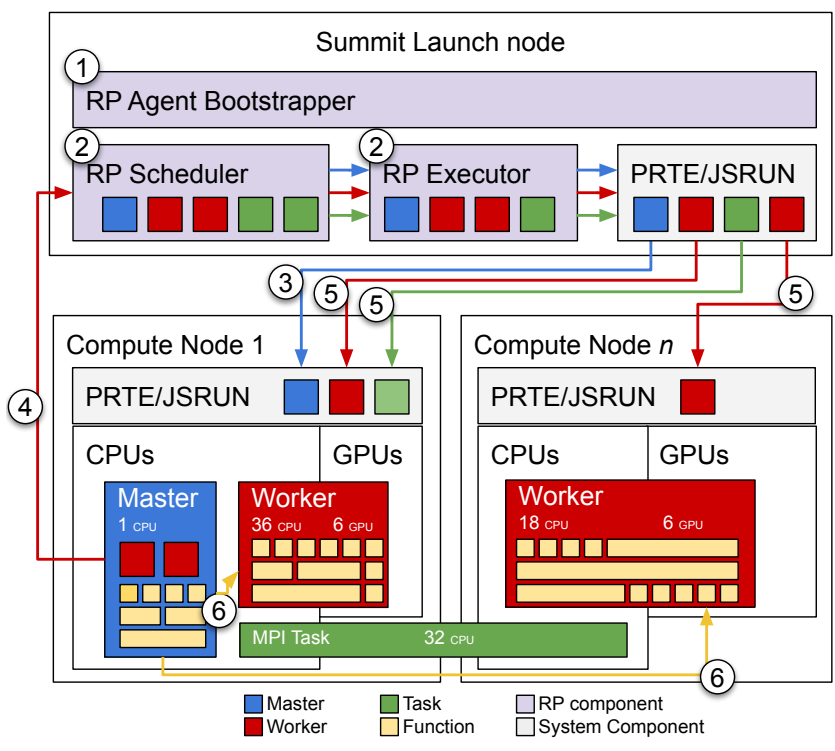

Figure 3: RAPTOR Execution Framework: One of the two execution frameworks used to support heterogeneous tasks and dynamic workloads on Summit.

SMILES string are gathered and concatenated on rank 0 and written into a CSV file which is forwarded to step S1.

6.1.2 S1: Physics-based Ensemble Docking To support the scaling requirements of S1, we implemented a Master/Worker overlay on top of the pilot-job abstraction. Fig. 3 illustrates the RAdicalPilot Task OveRlay (RAPTOR) master/worker implementation on Summit. Once RAPTOR has acquired its resources by submitting a job to Summit's batch system, it bootstraps its Agent (Fig. 3-1) and then launches a task scheduler and a task executor (Fig. 3-2). Scheduler and Executor launch one or more masters on one or more compute nodes (Fig. 3-3). Once running, a master schedules one or more workers on RP Scheduler (Fig. 3-4). Those workers are then launched on one or more compute nodes by RP Executor (Fig. 3-5). Finally, the master schedules function calls on the available workers for execution (Fig. 3-6), load-balancing across workers so to obtain maximal resource utilization.

The duration of the docking computation varies significantly between individual receptors. The long tail poses a challenge to load balancing; the relatively short docking times pose a challenge to scalability. Load balancing is addressed by iterating through the list of compounds in a round-robin fashion, and by dynamic load distribution which depends on the load of the individual workers. Further, balancing is achieved by: (i) tasks are communicated in bulks as to limit the communication load and frequency; (ii) multiple master processes are used to limit the number of workers served by each master, avoiding respective bottlenecks; (iii) multiple concurrent pilots are used to isolate the docking computation of individual compounds within each pilot allocation. The combination of these approaches results in a near linear scaling up to several thousand nodes, while maintaining high utilization for large numbers of concurrently used nodes. 


\subsubsection{S2 and S3: Advanced Sampling and Binding Free En-} ergy We implement $\mathrm{S} 2$ and $\mathrm{S} 3$ as iterative pipelines that comprise heterogeneous stages, with each stage supporting the parallel execution of tasks. In S2, the pipeline starts with MD simulations that are run concurrently; it completes a single iteration by passing through deep learning stages for AAE model training and the outlier detection. In a single iteration, tasks are scheduled across single GPU, multiple GPUs, and CPU-GPU tasks. For instance, the MD stage uses a single GPU per simulation (OpenMM), the data aggregation stage uses CPUs only, the ML training stage uses six GPUs per model, and the outlier detection stage uses a mixture of CPUs and GPUs. We also employ data parallelism for model training using PyTorch distributed data parallel module.

Similarly, S3 involves two stages of equilibration and one stage of simulation; each stage runs an ensemble of from six (S3-CG) to 24 (S3-FG) OpenMM tasks. We also employ NAMD-based TIES in conjunction with ESMACS; this requires placing distinct simulations to GPU (OpenMM) and CPU (NAMD) concurrently for the optimal resource utilization on Summit.

The architecture of RAPTOR (Fig. 3) differs from that of the classic RADICAL-Pilot used for S2 and S3 on Summit [45]. The need for two task execution frameworks arises primarily from the dynamism and heterogeneity of workloads. For example, six orders of magnitude difference in the temporal duration (Tab. 2) of tasks requires that the Master/Worker overlay sustain a throughput of up to $50 \mathrm{M}$ docking hits per hour on $\sim 1000$ nodes on Summit.

\section{Performance Results}

We report on both scientific and computational performance.

\subsection{Scientific Performance}

Available compound libraries are large, with ZINC providing over 230 million purchasable compounds [42] in ready-to-dock, 3D formats, and MCULE having 100 million purchasable compounds in similar formats [5]. Hence there is a need to obtain an appropriate sampling of the compound libraries based on the diversity of the compounds as well as their availability for both docking calculations (to generate the training data) and inference runs (ML1 results). We selected a subset of 6.5 million compounds from the ZINC library along with the Enamine diversity set [3] and DrugBank [52] compounds to develop our training library (OZD library, hereafter). We also chose a similar subset of 6.5 million compounds from the MCULE library (ORD library, hereafter) for the purposes of testing if ML1 can indeed be used for transferring knowledge learned from one library to another. These libraries pay attention only to the diversity of the compounds selected, and are independently selected, although between the two libraries we observed an overlap of approximately 1.5 million compounds.

7.1.1 ML1 results We trained our ML1 models on docking runs (generated offline) for the four main target SARS-CoV-2 proteins, namely 3C like protease (3CLPro), papain-like protease (PLPro), ADP-Ribose-1"-Monophosphatase (ADRP), and non-structural protein 15 (NSP15). These proteins all represent important drug targets against SARS-CoV-2 virus. Here we present only a vignette of results from the PLPro target and specifically from the receptor derived from the PDB-ID 6W9C. The RES plot from Fig. 4 indicates that the extraction of the top scoring molecules from the OZD library can be thought of as a selection process of $\delta$ molecules, to the subsequent

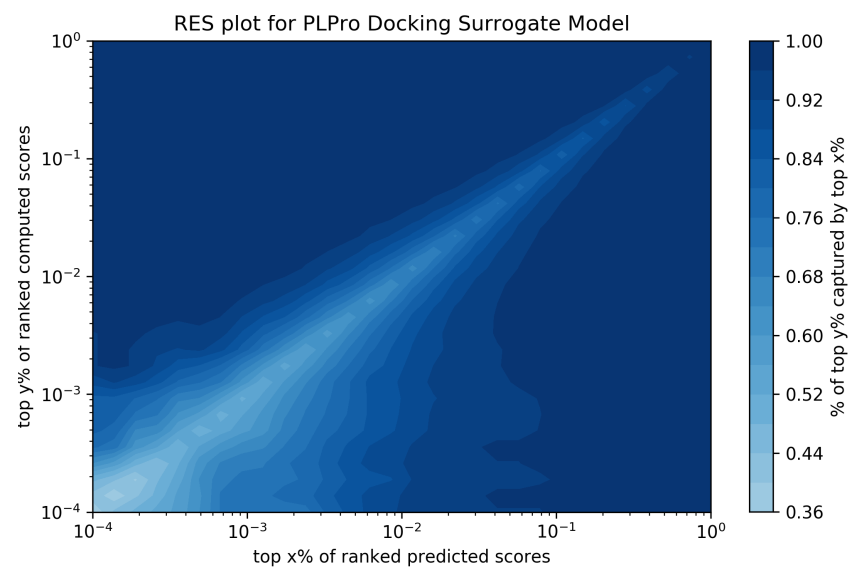

Figure 4: RES profile for PLPro docking runs. As explained in the main text, RES provides a summary estimate of how many top scoring compounds can be covered given some target number $(\delta)$ of molecules to be ranked.

stages. Given a specific budget of $\delta$ molecules to pass along, we can imagine a vertical line along the $x$-axis of Fig. 4 at the point $\delta / u$ representing the budget, where any point to the right of that line represents an unattainable number of compounds. One can also imagine a constraint through $y=x$, as points above this line represent situations where a wider range of the top distribution may prove too expensive, although reasonable for some tasks, uHTS screening is in pursuit of ultra high ranking compounds.

Given these two constraints, one can see that as $\delta$ increases, so to does the accuracy of capturing some desired threshold of the top distribution. If downstream tasks allot $\delta=u 10^{-3}$ compounds, then the plot indicates that we will capture $50 \%$ of the top ranking $u 10^{-4}$ compounds, or around $40 \%$ of the top ranking $u 10^{-3}$ compounds. In concrete terms, for this library, the ML model here correctly identifies 500 of the top 1000 scoring compounds from the docking study, or about 4000 of the top 10,000 compounds. However, not all top-ranking compounds are correlated with obtaining high binding affinity to PLPro. The RES plot also provides a quantitative estimate of the number of compounds we have to sample from lower ranking one so that we do not inadvertently miss out other high affinity compounds. Hence we also select about 15-20\% of compounds from the RES to the subsequent stages.

7.1.2 S3: CG-ESMACS For each target of the four target proteins mentioned above, multiple crystal structures were used to perform docking and a separate list of top 10,000 compounds based on their docking scores was generated at the ML1 stage. Therefore, depending on the number of crystal structures used for each target, there were collectively 20,000-40,000 compounds available for performing binding affinity predictions using CG-ESMACS. At this stage of our pipeline, we chose 10,000 compounds for each target by picking out the structurally most diverse compounds from all compounds available. This was done for two reasons: (i) based on the docking scores, all the available compounds were stable poses, and (ii) allowing for maximum possible coverage of the chemical space allowing for better and quicker identification of its relevant regions to focus on in next iterations. 


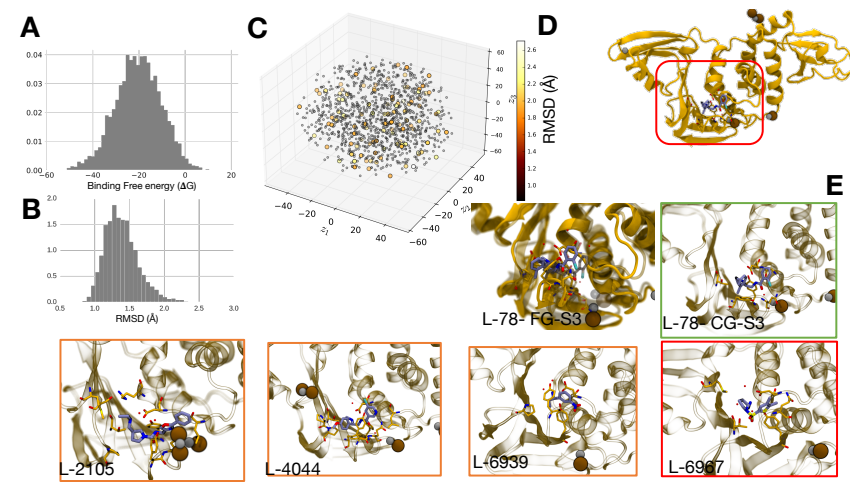

Figure 5: Preliminary results from IMPECCABLE on PLPro receptor (PDB ID: 6W9C). (A) Summary histogram of the distribution of binding free energies estimated using CGESMACS. (B) Summary of RMSD $(\AA)$ determined from CGESMACS LPC ensembles show a rather tight distribution with a few LPCs that exhibit greater fluctuations. (C) Latent space representation from the 3D-AAE model depicting the outliers from RMSD distributions ( $>1.9$ ) and the rest as gray dots. The latent space also summarizes the extent of sampling from these simulations. (D) Structure of PLPro bound to one of the highly specific molecule (L78) in its active site. (E) A zoomed in version of the same compound (L78) showing close interactions with key residues in PLPro (green highlight). The panel on the left depicts how upon running FG-ESMACS we obtain tighter binding through the compound moving further into the binding site, forming strong hydrophobic interactions and hydrogen bonds. Other compounds that did not perform as well in the FG-ESMACS (see 6) are shown for comparison; although the bound structures exhibit similar interaction patterns, none of them stabilize further in the binding site, leading to a reduction in their binding free energy estimates.

We performed CG-ESMACS to get binding affinities for all these compounds chosen, amounting to a total of 40,000 S3-CG calculations thus far. Fig. 5A shows a probability distribution of the 10,000 binding affinities for PLPro. The values typically lie between -60 to $+20 \mathrm{kcal} / \mathrm{mol}$ for all proteins. The resultant trajectories and binding affinity values from this stage were used as input for S2 to identify potentially useful conformations that were fed into S3-FG.

7.1.3 Using S2 to seed S3-FG For PLPro, about 5000 compounds were chosen based on the structural diversity criterion for PDBID 6w9c. The trajectories corresponding to these 5000 compounds generated by S3-CG were used to build a combined dataset of 100,978 examples. The point cloud data, representing the coordinates of the 309 backbone $\mathrm{C}^{\alpha}$ atoms of the protein, was randomly split into training $(80 \%)$ and validation input $(20 \%)$ and was used to train the 3D-AAE model for 100 epochs using a batch size of 64 . The data was projected onto a latent space of 64 dimensions constrained by a Gaussian prior distribution with a standard deviation of 0.2 . The loss optimization was performed with the Root Mean Square Propagation (RMSprop) optimizer, a gradient descent algorithm for mini-batch learning, using a learning rate of 0.00001 .
We also added hyperparameters to scale individual components of the loss. The reconstruction loss was scaled by 0.5 and the gradient penalty was scaled by a factor of 10 . We trained the model using several combinations of hyperparameters, mainly varying learning rate, batch size and latent dimension. The embedding learned from the 3D-AAE model summarizes a latent space that is similar to variational autoencoders, except that 3D-AAEs tend to be more robust to outliers within the simulation data. The embeddings learned from the simulations allow us to cluster the conformations (in an unsupervised manner) based on their similarity in overall structure, which can be typically measured using quantities such as root-mean squared deviations (RMSD). The 5,000 ligands were further analyzed and 5 structures with the lowest free energy (L6967, L2105, L78, L6939, L4044) were selected for generating embeddings for 1200 examples, using the hyperparameters learned from 3D-AAE performed on the full set of 5,000 ligands. For visualizing and assessing the quality of the model in terms latent space structure, we computed t-stochatstic neighborhood embedding (t-SNE) [30] on the embeddings from the validation set. The validation data was painted with grey while the test data was painted with the root mean squared deviation (RMSD) of each structure to the starting conformation (Fig. 5B-C).

7.1.4 S3: FG-ESMACS The large amount of data generated by S3-CG was analysed at S2 and based on that potentially good conformations were identified for the compounds with large negative binding affinities from CG-ESMACS. This process led us to filter out five outlier conformations each for the top five compounds based on S3-CG results. We used these 25 conformations to perform the costlier FG-ESMACS calculations to demonstrate the capability of our pipeline to confidently identify favourable interactions between protein and ligands. This helps us mark favourable regions in the chemical space deserving more attention, which in turn trains our ML model to generate and/or predict better compounds in the next iteration. Fig. 6 displays a comparison of the provisional results from FG-ESMACS with those from CG-ESMACS. It is manifest that FG-ESMACS predicts much lower binding affinities than those predicted by CG-ESMACS (Fig. 5D-E). The force-field used in both cases was the same; only the starting structures varied between them. This implies that the outliers filtered out by S2 indeed captured some favourable interactions and successfully identified good conformations out of the large number of conformations generated by S3-CG. This is an excellent demonstration of the novel capability of IMPECCABLE to quickly sample the relevant chemical space and hence accelerate the process of drug discovery. With every iteration, such refinements improve the compounds generated by our ML model manifold: we expect to design potential inhibitors for the target protein in much less time than the standard drug discovery approaches.

\subsection{Computational Performance}

Fig. 7 shows an example of how independent pipelines can be integrated into a single workflow. Each pipeline is comprised of stages, each with an arbitrary number of tasks. Tasks have heterogeneous execution time and computational requirements. Stages can execute concurrently or sequentially, depending on available resources and task, stage and pipeline interdependencies. In the depicted integration, single-GPU tasks execute alongside MPI GPU and few CPU tasks, in distinct and customized execution environments. Note that 


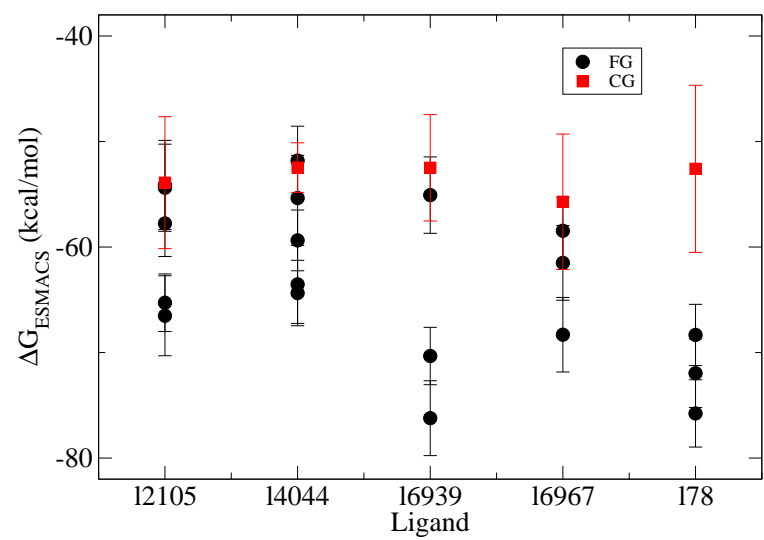

Figure 6: Comparison of S3-CG and S3-FG results for the five best binders for PLPro (PDBID: 6w9c) based on CG-ESMACS results. $\mathrm{S} 2$ selected five outlier conformations for each binder and performed FG-ESMACS on them. The provisional results confirm improved binding for the selected conformations in all five compounds, as FG energies are lower than CG.

the overheads (light-colored vertical areas of the plots) are invariant to scale, i.e., they do not depend on the number of concurrent tasks executed or on the length of those tasks.

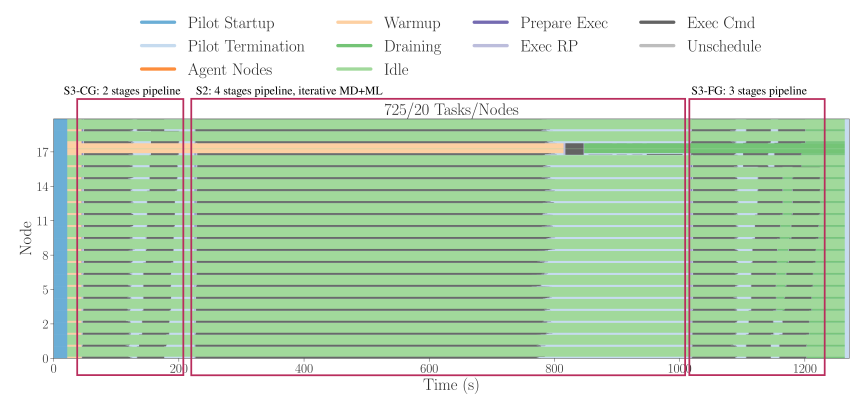

Figure 7: A time-series of node utilization. The Fig. depicts the integrated execution of three GPU-intensive workflows (S3CG)-(S2)-(S3-FG). S3-CG, S2 and S3-FG are heterogeneous and multi-stage workflows themselves.

We measure flops (floating point operations, not rates) per work unit for the most relevant components of each stage. We define a work unit to be a representative code section such as an MD time integration step for MD-based or a data sample for DL-based applications. Thus we can compute the aggregate invested flops by scaling the measured flop counts to the respective work set sizes used in the actual runs.

Table 3: Throughput and performance measured as peak flop per second (mixed precision, measured over short but time interval) per Summit node (6 NVIDIA V100 GPU).

\begin{tabular}{c|rrr}
\hline Comp. & \#GPUs & Tflop/s & Throughput \\
\hline \hline ML1 & 1536 & 753.9 & 319674 ligands/s \\
S1 & 6000 & 112.5 & 14252 ligands/s \\
S3-CG & 6000 & 277.9 & 2000 ligand/s \\
S3-FG & 6000 & 732.4 & 200 ligand/s \\
\hline
\end{tabular}

We always normalize the measurements to a single Summit node for the same reason. As all of our applications are perfectly load balanced with respect to a Summit node (mostly even with respect to individual GPUs within that node), this procedure yields a representative flop count. We use the methodology of Yang et al. [59] and the NVIDIA NSight Compute 2020 GPU profiling tool to measure flops for all precisions and sum them to obtain a mixed precision flop count. When possible, we use start/stop profiler hooks to filter out the representative work units. In order to obtain the flop rate, we divide the aggregated flops for each EnTK task by the time it takes to complete that respective task, including pre- and post-processing overhead. Note that we do not account for any CPU flops invested in this calculation as we expect that number to be small. We discuss the specifics for each component:

ML1: We count flops as described above for 10 steps at batch size 256. From that, we derive a flop count per batch per GPU.

S1: We count flops for a five-ligand AutoDock-GPU run on one GPU to derive flops for a single ligand. We chose this ligand complexity to represent the majority of the ligands processed in the run.

S2: This stage has multiple steps, but we only account for the autoencoder training and the MD performed in this stage. For the former, we measure the flops per batch for a batch size of 32 for training and validation separately and weight them proportionally by their relative number of batches. After each training epoch, a validation is performed and the train/validation dataset split is $80 \% / 20 \%$. This can be translated into a overall flop count for the full autoencoder stage. For the MD part, we profile 20 steps of OpenMM and compute a complexity per step.

S3-CG/FG: These two stages both have two steps, a minimization and an MD step. We count the flops for 10 iterations of the minimization algorithm and for 20 steps of the MD run to derive a flop count per minimization and MD step. Since the algorithmic complexity differs between CG and FG, we profile those separately.

\section{Implications}

Multiscale biophysics-based computational lead discovery is an important strategy for drug development and while it can be considerably faster than experimental screening it has been until now too slow to explore libraries of the scale of billions of molecules even on the fastest machines.

The work reported here not only addresses this performance issue, by demonstrating a path towards an overall improvement of throughput of computational drug discovery of order 1000x by integrating machine learning components with the physics based components, but it also addresses other important aspects of improved workflows for computational lead discovery, namely the generalization and integration of feedback between the physics models and the ML models in a tightly coupled workflow.

By introducing ML modules paired with and trained from the physics modules output, over time the ML component models improve such that the overall workflow becomes tuned to the specific target problem. It is also likely that it will be possible to transfer these learnings from one problem to the next. The generalizability of this approach is under active investigation.

The feedback is not only local to each stage of the workflow but end-to-end, so this work also represents the beginning of an 
autonomous drug development system when coupled to automated experimental screening and eventually to drug synthesis capability.

The work reported here has played a central role in the DOE effort to use computational molecular design approaches to develop medical therapeutics for COVID-19. DOE established in April 2020 the National Virtual Biotechnology Laboratory (NVBL) [4], to organize the DOE national laboratories into a series of projects-including therapeutics development-aimed at addressing key challenges in responding to COVID-19. Methods and infrastructure reported in this paper are being used to screen over 4.2 billion molecules [10] against over a dozen drug targets in SARS-CoV-2. This work has already lead to the identification and experimental validation of over 1000 compounds, resulting in over 40 hits that are progressing to advanced testing.

To get a sense of the scale of operations using methods and infrastructure reported here: in the past three months, we have used more than 2.5M node-hours across diverse HPC platforms, including TACC's Frontera, Livermore Computing's Lassen, ANL's Theta (and associated A100 nodes), LRZ's SuperMUC-NG, and ORNL Summit to obtain scientific results. We have executed individual parts of the campaign on suitable platforms, e.g., we sustained $40 \mathrm{M}$ docking hits per hour over 24 hours on 4000 nodes on Frontera. We performed thousands of AI-driven MD simulations across GPUbased systems: Lassen, Summit and Theta's A100 nodes, as well as a few tens of thousands of CG-ESMACS runs on Summit, ARCHER (EPCC, UK), Monsoon2 (Metoffice, UK) and SuperMUC-NG.

In doing so, IMPECCABLE has screened $\sim 10^{11}$ ligands. We have performed up to $5 \times 10^{7}$ docking-hits per hour using OpenEye and Autodock-GPU, and sustained this throughput on $\sim 4000$ nodes. Individual workflow components deliver $100 \times$ to $1000 \times$ improvement over traditional methods. IMPECCABLE has computed binding free energies on $10^{4}$ LPCs concurrently.

While much work remains to be done, we have demonstrated some important milestones towards the ultimate goal. These include: orders of magnitude end-to-end performance improvement of computational methods for drug discovery, making it feasible and routine to search giga-scale libraries of compounds across collections of drug targets; integration of physics based modeling with AI methods into multiscale workflow at the largest-scale possible providing a pathway for exascale drug discovery, and developed a prototype infrastructure that can be adapted to a broad range of near autonomous drug development scenarios by means of additional modules and models that fill out the drug discovery pipeline.

We have developed this campaign with multiple target problems in mind. A major influence is our experience in working on the DOE/NCI JDAS4C Pilot1 effort to advance Cancer drug development through AI [58] and the related ECP CANDLE project [53]. In the Pilot 1 activity we are building Cancer drug response models that predict the response of tumors to drugs or drug combinations. Such models could be coupled to the the workflow described in this paper to add additional feedback on predicted efficacy of a target molecule. Work in this area is ongoing to build a bridge between tissue level efficacy models such as the ones developed in Pilot 1 and CANDLE and drug target oriented models reported here.

We envision ultimately a series of models to be part of this extended workflow that predict not only efficacy, but also other important properties of candidate compounds such as drug metabolism, pharmacokinetics, absorption, toxicity, distribution, and excretion that traditionally would be assessed via experimental methods. Building a comprehensive suite of ML-based predictors is precisely the goal of the ATOM consortium, to which this project is a contributor. ATOM aims to dramatically accelerate drug development by building and training ML models that systematically replace routine experimental screening in early stages of drug development, with the goal of reducing the time from drug target to clinical trial from 5-6 years to one year.

Acknowledgements: Research was supported by the DOE Office of Science through the National Virtual Biotechnology Laboratory, a consortium of DOE national laboratories focused on response to COVID-19, with funding provided by the Coronavirus CARES Act. This research was supported as part of the CANDLE project by the Exascale Computing Project (17-SC20-SC), a collaborative effort of the U.S. Department of Energy Office of Science and the National Nuclear Security Administration. This work has been supported in part by the Joint Design of Advanced Computing Solutions for Cancer (JDACS4C) program established by the U.S. Department of Energy (DOE) and the National Cancer Institute (NCI) of the National Institutes of Health. We are grateful for funding for the UK MRC Medical Bioinformatics project (grant no. MR/L016311/1), the UK Consortium on Mesoscale Engineering Sciences (UKCOMES grant no. EP/L00030X/1) and the European Commission for the EU H2020 CompBioMed2 Centre of Excellence (grant no. 823712), as well as financial support from the UCL Provost. Access to SuperMUC-NG, at the Leibniz Supercomputing Centre in Garching, was made possible by a special COVID-19 allocation award from the Gauss Centre for Supercomputing in Germany. Anda Trifan acknowledges support from the United States Department of Energy through the Computational Sciences Graduate Fellowship (DOE CSGF) under grant number: DE-SC0019323. We acknowledge amazing support from OLCFDon Maxwell, Bronson Messier and Sean Wilkinson. We also wish to thank Dan Stanzione and Jon Cazes at Texas Advanced Computing Center. 


\section{References}

[1] [n. d.]. COVID-19 Molecular Structure and Therapeutics Hub. https://covid. molssi.org.

[2] [n. d.]. COVID Moonshot. https://covid.postera.ai/covid.

[3] [n. d.]. Enamine diversity library. https://enamine.net/hit-finding/diversitylibraries.

[4] [n. d.]. National Virtual Biotechnology Laboratory (NVBL). https://science.osti. gov/nvbl.

[5] [n. d.]. Ultimate 100 Million Compounds. https://ultimate.mcule.com.

[6] 2020. NVIDIA TensorRT developer blog. https://developer.nvidia.com/blog/tag/ tensorrt/

[7] 2020. rdKit Mol2D drawing documentation. http://rdkit.org/docs/source/rdkit Chem.Draw.rdMolDraw2D.html

[8] 2020. torch2trt github repository. https://github.com/NVIDIA-AI-IOT/torch2trt

[9] Dinler A Antunes, Didier Devaurs, and Lydia E Kavraki. 2015. Understanding the challenges of protein flexibility in drug design. Expert opinion on drug discovery 10, 12 (2015), 1301-1313.

[10] Yadu Babuji, Ben Blaiszik, Tom Brettin, Kyle Chard, Ryan Chard, Austin Clyde, Ian Foster, Zhi Hong, Shantenu Jha, Zhuozhao Li, et al. 2020. Targeting SARSCoV-2 with AI-and HPC-enabled lead generation: A First Data release. arXiv preprint arXiv:2006.02431 (2020).

[11] Yadu Babuji, Anna Woodard, Zhuozhao Li, Daniel S Katz, Ben Clifford, Rohan Kumar, Lukasz Lacinski, Ryan Chard, Justin M Wozniak, Ian Foster, and Kyle Chard. 2019. Parsl: Pervasive parallel programming in Python. In 28th International Symposium on High-Performance Parallel and Distributed Computing. 25-36.

[12] Vivek Balasubramanian, Matteo Turilli, Weiming Hu, Matthieu Lefebvre, Wen jie Lei, Ryan Modrak, Guido Cervone, Jeroen Tromp, and Shantenu Jha. 2018 Harnessing the power of many: Extensible toolkit for scalable ensemble applications. In 2018 IEEE International Parallel and Distributed Processing Symposium (IPDPS). IEEE, 536-545.

[13] Pedro J. Ballester, Adrian Schreyer, and Tom L. Blundell. 2014. Does More Precise Chemical Description of Protein-Ligand Complexes Lead to More Accurate Prediction of Binding Affinity? Journal of Chemical Information and Modeling 54, 3 (2014), 944-955. https://doi.org/10.1021/ci500091r arXiv:https://doi.org/10.1021/ci500091r PMID: 24528282.

[14] Debsindhu Bhowmik, Shang Gao, Michael T. Young, and Arvind Ramanathan 2018. Deep clustering of protein folding simulations. BMC Bioinformatics 19,18 (2018), 484. https://doi.org/10.1186/s12859-018-2507-5

[15] Regine S Bohacek, Colin McMartin, and Wayne C Guida. 1996. The art and practice of structure-based drug design: A molecular modeling perspective. Medicinal research reviews 16, 1 (1996), 3-50.

[16] JR Broach and J Thorner. 1996. High-throughput screening for drug discovery. Nature 384, 6604 Suppl (November 1996), 14-16. https://doi.org/10.1038/ $384014 \mathrm{a} 0$

[17] Sharan Chetlur, Cliff Woolley, Philippe Vandermersch, Jonathan Cohen, John Tran, Bryan Catanzaro, and Evan Shelhamer. 2014. cuDNN: Efficient Primitives for Deep Learning. arXiv:cs.NE/1410.0759

[18] Austin Clyde, Xiaotian Duan, and Rick Stevens. 2020. Regression Enrichment Surfaces: a Simple Analysis Technique for Virtual Drug Screening Models. arXiv preprint arXiv:2006.01171 (2020).

[19] Jumana Dakka, Kristof Pallas Vivek Balasubramanian, Matteo Turilli, David W Wright, Stefan J Zasada, Shunzhou Wan, Peter V Coveney, and Shantenu Jha. 2018. Concurrent and Adaptive Extreme Scale Binding Free Energy Calculations. 14th IEEE International Conference on e-Science, e-Science 2018, Amsterdam (2018), 189-200. https://doi.org/10.1109/eScience.2018.00034

[20] Jumana Dakka, Kristof Farkas-Pall, Vivek Balasubramanian, Matteo Turilli, Shunzhou Wan, David W. Wright, Stefan J. Zasada, Peter V. Coveney, and Shantenu Jha. 2018. Enabling Trade-offs Between Accuracy and Computational Cost: Adaptive Algorithms to Reduce Time to Clinical Insight. In 18th IEEE/ACM International Symposium on Cluster, Cloud and Grid Computing, CCGRID 2018, Washington, DC, USA, May 1-4, 2018. 572-577. https://doi.org/10.1109/CCGRID.2018.00005

[21] Daniel C. Elton, Zois Boukouvalas, Mark D. Fuge, and Peter W. Chung. 2019 Deep learning for molecular design - a review of the state of the art. Molecular Systems Design \& Engineering 4, 4 (2019), 828-849. https://doi.org/10.1039/ c9me00039a

[22] Garrett B. Goh, Charles Siegel, Abhinav Vishnu, Nathan O. Hodas, and Nathan Baker. 2017. Chemception: A Deep Neural Network with Minimal Chemistry Knowledge Matches the Performance of Expert-developed QSAR/QSPR Models. arXiv:stat.ML/1706.06689

[23] Isabella A Guedes, Felipe SS Pereira, and Laurent E Dardenne. 2018. Empirical scoring functions for structure-based virtual screening: applications, critical aspects, and challenges. Frontiers in Pharmacology 9 (2018), 1089

[24] Kaiming He, Xiangyu Zhang, Shaoqing Ren, and Jian Sun. 2015. Deep Residual Learning for Image Recognition. arXiv:cs.CV/1512.03385

[25] James P Hughes, Stephen Rees, S Barrett Kalindjian, and Karen L Philpott. 2011. Principles of early drug discovery. British journal of pharmacology 162, 6 (2011),
$1239-1249$.

[26] Ashutosh Kumar and Kam Y.J. Zhang. 2015. Hierarchical virtual screening approaches in small molecule drug discovery. Methods 71 (2015), 26 - 37. https://doi.org/10.1016/j.ymeth.2014.07.007 Virtual Screening.

[27] Yann LeCun, Yoshua Bengio, and Geoffrey Hinton. 2015. Deep learning. Nature 521, 7553 (2015), 436-444. https://doi.org/10.1038/nature14539

[28] H. Lee, M. Turilli, S. Jha, D. Bhowmik, H. Ma, and A. Ramanathan. 2019. DeepDriveMD: Deep-Learning Driven Adaptive Molecular Simulations for Protein Folding. In 2019 IEEE/ACM Third Workshop on Deep Learning on Supercomputers $(D L S)$. 12-19.

[29] Hyungro Lee, Matteo Turilli, Shantenu Jha, Debsindhu Bhowmik, Heng Ma, and Arvind Ramanathan. 2019. DeepDriveMD: Deep-Learning Driven Adaptive Molecular Simulations for Protein Folding. In 2019 IEEE/ACM Third Workshop on Deep Learning on Supercomputers (DLS). IEEE, 12-19. https://doi.org/10. 1109/DLS49591.2019.00007 arXiv:1909.07817

[30] Laurens van der Maaten and Geoffrey Hinton. 2008. Visualizing data using t-SNE. Journal of machine learning research 9, Nov (2008), 2579-2605.

[31] Andre Merzky, Matteo Turilli, Manuel Maldonado, Mark Santcroos, and Shantenu Jha. 2018. Using pilot systems to execute many task workloads on supercomputers. In Workshop on Job Scheduling Strategies for Parallel Processing. Springer, 6182.

[32] Amanda J Minnich, Kevin McLoughlin, Margaret Tse, Jason Deng, Andrew Weber, Neha Murad, Benjamin D Madej, Bharath Ramsundar, Tom Rush, Stacie Calad-Thomson, et al. 2020. AMPL: A Data-Driven Modeling Pipeline for Drug Discovery. Journal of Chemical Information and Modeling 60, 4 (2020), 19551968.

[33] N Moitessier, P Englebienne, D Lee, J Lawandi, and C R Corbeil. 2008. Towards the development of universal, fast and highly accurate docking/scoring methods: a long way to go. British Journal of Pharmacology 153, S1 (2008), S7-S26. https://doi.org/10.1038/sj.bjp.0707515 arXiv:https://bpspubs.onlinelibrary.wiley.com/doi/pdf/10.1038/sj.bjp.0707515

[34] Jerry M. Parks and Jeremy C. Smith. 2020. How to Discover Antiviral Drugs Quickly. New England Journal of Medicine 382, 23 (2020), 2261-2264. https://doi.org/10.1056/NEJMcibr2007042 arXiv:https://doi.org/10.1056/NEJMcibr2007042 PMID: 32433861.

[35] Bharath Ramsundar, Steven Kearnes, Patrick Riley, Dale Webster, David Konerding, and Vijay Pande. 2015. Massively Multitask Networks for Drug Discovery. arXiv:stat.ML/1502.02072

[36] Research in Advanced DIstributed Cyberinfrastructure and Applications Laboratory (RADICAL). 2019. RADICAL-Cybertools (RCT). https://github.com/ radical-cybertools, Last accessed on 2019-03-31.

[37] Raquel Romero, Arvind Ramanathan, Tony Yuen, Debsindhu Bhowmik, Mehr Mathew, Lubna Bashir Munshi, Seher Javaid, Madison Bloch, Daria Lizneva, Alina Rahimova, Ayesha Khan, Charit Taneja, Se-Min Kim, Li Sun, Maria I. New, Shozeb Haider, and Mone Zaidi. 2019. Mechanism of glucocerebrosidase activation and dysfunction in Gaucher disease unraveled by molecular dynamics and deep learning. Proceedings of the National Academy of Sciences 116, 11 (2019), 5086-5095. https://doi.org/10.1073/pnas.1818411116 arXiv:https://www.pnas.org/content/116/11/5086.full.pdf

[38] S. Kashif Sadiq, David Wright, Simon J. Watson, Stefan J. Zasada, Ileana Stoica, and Peter V. Coveney. 2008. Automated Molecular Simulation Based Binding Affinity Calculator for Ligand-Bound HIV-1 Proteases. Journal of Chemical Information and Modeling 48, 9 (2008), 1909-1919. https://doi.org/10.1021/ ci8000937 arXiv:https://doi.org/10.1021/ci8000937 PMID: 18710212.

[39] Atanu Saha and Heather Roberts. 2020. Pharmaceutical industry's changing market dynamics. International Journal of the Economics of Business (2020), $1-17$.

[40] Justin S Smith, Adrian E Roitberg, and Olexandr Isayev. 2018. Transforming computational drug discovery with machine learning and AI.

[41] Francesca Spyrakis, Paolo Benedetti, Sergio Decherchi, Walter Rocchia, Andrea Cavalli, Stefano Alcaro, Francesco Ortuso, Massimo Baroni, and Gabriele Cruciani. 2015. A Pipeline To Enhance Ligand Virtual Screening: Integrating Molecular Dynamics and Fingerprints for Ligand and Proteins. Journal of Chemical Information and Modeling 55, 10 (2015), 2256-2274. https://doi.org/10. 1021/acs.jcim.5b00169 arXiv:https://doi.org/10.1021/acs.jcim.5b00169 PMID: 26355717

[42] Teague Sterling and John J. Irwin. 2015. ZINC 15 - Ligand Discovery for Everyone. Journal of Chemical Information and Modeling 55, 11 (2015), 2324-2337. https://doi.org/10.1021/acs.jcim.5b00559 arXiv:https://doi.org/10.1021/acs.jcim.5b00559 PMID: 26479676.

[43] T. P. Straatsma, H. J. C. Berendsen, and J. P. M. Postma. 1986. Free energy of hydrophobic hydration: A molecular dynamics study of noble gases in water. The Journal of Chemical Physics 85, 11 (1986), 6720-6727. https://doi.org/10.1063/ 1.451846 arXiv:https://doi.org/10.1063/1.451846

[44] Matteo Turilli, Vivek Balasubramanian, Andre Merzky, Ioannis Paraskevakos, and Shantenu Jha. 2019. Middleware building blocks for workflow systems. Computing in Science \& Engineering 21, 4 (2019), 62-75. 
[45] Matteo Turilli, Andre Merzky, Thomas Naughton, Wael Elwasif, and Shantenu Jha. 2019. Characterizing the Performance of Executing Many-tasks on Summit. IPDRM Workshop, SC19 (2019). https://arxiv.org/abs/1909.03057.

[46] Matteo Turilli, Mark Santcroos, and Shantenu Jha. 2018. A Comprehensive Perspective on Pilot-Job Systems. ACM Comput. Surv. 51, 2, Article 43 (April 2018), 32 pages. https://doi.org/10.1145/3177851

[47] Shunzhou Wan, Agastya P. Bhati, Sarah Skerratt, Kiyoyuki Omoto, Veerabahu Shanmugasundaram, Sharan K. Bagal, and Peter V. Coveney. 2017. Evaluation and Characterization of Trk Kinase Inhibitors for the Treatment of Pain: Reliable Binding Affinity Predictions from Theory and Computation. Journal of Chemical Information and Modeling 57, 4 (2017), 897-909. https://doi.org/10.1021/acs jcim.6b00780 arXiv:https://doi.org/10.1021/acs.jcim.6b00780 PMID: 28319380

[48] Shunzhou Wan, Agastya P Bhati, Stefan J Zasada, and Peter V Coveney. 2020. Rapid, accurate, precise and reproducible ligand-protein binding free energy prediction. Journal of Royal Society Interface Focus 10 (2020). https://doi.org/ 10.1098/rsfs.2020.0007

[49] Shunzhou Wan, Agastya P. Bhati, Stefan J. Zasada, Ian Wall, Darren Green, Paul Bamborough, and Peter V. Coveney. 2017. Rapid and Reliable Binding Affinity Prediction of Bromodomain Inhibitors: A Computational Study. Journal of Chemical Theory and Computation 13, 2 (2017), 784-795. https://doi.org/ 10.1021/acs.jctc.6b00794 arXiv:https://doi.org/10.1021/acs.jctc.6b00794 PMID 28005370.

[50] Shunzhou Wan, Bernhard Knapp, David W. Wright, Charlotte M. Deane, and Peter V. Coveney. 2015. Rapid, Precise, and Reproducible Prediction of Peptide-MHC Binding Affinities from Molecular Dynamics That Correlate Well with Experiment. Journal of Chemical Theory and Computation 11, 7 (2015), 3346-3356. https://doi.org/10.1021/acs.jctc.5b00179 arXiv:https://doi.org/10.1021/acs.jctc.5b00179 PMID: 26575768.

[51] Xueqing Wang and Yuanfang Guan. [n. d.]. COVID-19 drug repurposing: A review of computational screening methods, clinical trials, and protein interaction assays. Medicinal Research Reviews n/a, n/a ([n. d.]). https://doi.org/10.1002/med.21728 arXiv:https://onlinelibrary.wiley.com/doi/pdf/10.1002/med.21728

[52] David S Wishart, Yannick D Feunang, An C Guo, Elvis J Lo, Ana Marcu, Jason R Grant, Tanvir Sajed, Daniel Johnson, Carin Li, Zinat Sayeeda, Nazanin Assempour, Ithayavani Iynkkaran, Yifeng Liu, Adam Maciejewski, Nicola Gale, Alex Wilson, Lucy Chin, Ryan Cummings, Diana Le, Allison Pon, Craig Knox, and Michael Wilson. 2017. DrugBank 5.0: a major update to the DrugBank database for 2018. Nucleic Acids Research 46, D1 (11 2017), D1074-D1082. https://doi.org/10.1093/nar/gkx1037 arXiv:https://academic.oup.com/nar/articlepdf/46/D1/D1074/23162116/gkx1037.pdf

[53] Justin M. Wozniak, Rajeev Jain, Prasanna Balaprakash, Jonathan Ozik, Nicholson T. Collier, John Bauer, Fangfang Xia, Thomas Brettin, Rick Stevens, Jamaludin Mohd-Yusof, Cristina Garcia Cardona, Brian Van Essen, and Matthew Baughman. 2018. CANDLE/Supervisor: A workflow framework for machine learning applied to cancer research. BMC Bioinformatics 19, 491 (2018).

[54] David W. Wright, Benjamin A. Hall, Owain A. Kenway, Shantenu Jha, and Peter V. Coveney. 2014. Computing Clinically Relevant Binding Free Energies of HIV-1 Protease Inhibitors. Journal of Chemical Theory and Computation 10, 3 (2014), 1228-1241. https://doi.org/10.1021/ct4007037 arXiv:https://doi.org/10.1021/ct4007037 PMID: 24683369.

[55] David W. Wright, Fouad Husseini, Shunzhou Wan, Christophe Meyer, Herman van Vlijmen, Gary Tresadern, and Peter V. Coveney. 2020. Application of the ESMACS Binding Free Energy Protocol to a Multi-Binding Site Lactate Dehydogenase A Ligand Dataset. Advanced Theory and Simulations 3, 1 (2020), 1900194. https://doi.org/10.1002/adts.201900194 arXiv:https://onlinelibrary.wiley.com/doi/pdf/10.1002/adts.201900194

[56] David W Wright, Shunzhou Wan, Christophe Meyer, Herman Van Vlijmen, Gary Tresadern, and Peter V Coveney. 2019. Application of ESMACS binding free energy protocols to diverse datasets: Bromodomain-containing protein 4. Scientific Reports 9, 1 (2019), 1-15.

[57] Zhenqin Wu, Bharath Ramsundar, Evan N. Feinberg, Joseph Gomes, Caleb Geniesse, Aneesh S. Pappu, Karl Leswing, and Vijay Pande. 2018. MoleculeNet: a benchmark for molecular machine learning. Chem. Sci. 9 (2018), 513-530. Issue 2. https://doi.org/10.1039/C7SC02664A

[58] Fangfang Xia, Maulik Shukla, Thomas Brettin, Cristina Garcia-Cardona, Judith Cohn, Jonathan E. Allen, Sergei Maslov, Susan L. Holbeck, James H. Doroshow, Yvonne A. Evrard, Eric A. Stahlberg, and Rick L. Stevens. 2018. Predicting tumor cell line response to drug pairs with deep learning 19 (2018).

[59] Charlene Yang. 2020. Hierarchical Roofline Analysis: How to Collect Data using Performance Tools on Intel CPUs and NVIDIA GPUs. arXiv:cs.DC/2009.02449

[60] Xiaohua Zhang, Sergio E. Wong, and Felice C. Lightstone. 2014. Toward Fully Automated High Performance Computing Drug Discovery: A Massively Parallel Virtual Screening Pipeline for Docking and Molecular Mechanics/Generalized Born Surface Area Rescoring to Improve Enrichment. Journal of Chemical Information and Modeling 54, 1 (2014), 324-337. https://doi.org/10.1021/ci4005145 arXiv:https://doi.org/10.1021/ci4005145 PMID: 24358939.

[61] Yadi Zhou, Fei Wang, Jian Tang, Ruth Nussinov, and Feixiong Cheng. 2020. Artificial intelligence in COVID-19 drug repurposing. The Lancet Digital Health
(2020).

[62] Robert W. Zwanzig. 1954. High-Temperature Equation of State by a Perturbation Method. I. Nonpolar Gases. The Journal of Chemical Physics 22, 8 (1954), 14201426. https://doi.org/10.1063/1.1740409 arXiv:https://doi.org/10.1063/1.1740409

[63] Johan Åqvist, Victor B. Luzhkov, and Bjørn O. Brandsdal. 2002. Ligand Binding Affinities from MD Simulations. Accounts of Chemical Research 35, 6 (2002), 358365. https://doi.org/10.1021/ar010014p arXiv:https://doi.org/10.1021/ar010014p PMID: 12069620. 